\title{
SHAPE THEORY AND COMPACT CONNECTED ABELIAN TOPOLOGICAL GROUPS(1)
}

\author{
BY \\ JAMES KEESLING
}

\begin{abstract}
Let $C$ denote the category of compact Hausdorff spaces and continuous maps. Let $S: C \rightarrow S C$ denote the functor of shape in the sense of Holsztyński from $C$ to the shape category $S C$ determined by the homotopy functor $H: C \rightarrow H C$ from $C$ to the homotopy category $H C$. Let $A, B$, and $D$ denote compact connected abelian topological groups. In this paper it is shown that if $G$ is a morphism in the shape category from $A$ to $B$, then there is a unique continuous homomorphism $g: A \rightarrow B$ such that $S(g)=G$. This theorem is used in a study of shape properties of continua which support an abelian topological group structure. The following results are shown: (1) The spaces $A$ and $B$ are shape equivalent if and only if $A \simeq B$. (2) The space $A$ is movable if and only if $A$ is locally connected. (3) The space $A$ shape dominates $B, S(A) \geq S(B)$, if and only if there is a $D$ such that $A \simeq B \times D$. (4) The fundamental dimension of $A$ is the same as the dimension of $A, \operatorname{Sd}(A)=\operatorname{dim} A$.

In an Appendix it is shown that the Holsztyniski approach to shape and the approach of Mardešic and Segal using ANR-systems are equivalent. Thus, the results apply to either theory and to the Borsuk theory in the metrizable case.
\end{abstract}

Introduction. Let $C$ denote the category of compact Hausdorff spaces and continuous maps. Let $S: C \rightarrow S C$ denote the functor of shape in the sense of Holsztyński on the category $C$ of compact Hausdorff spaces and continuous maps to the shape category $S C$ (see [6, Example 5.9]). This functor is continuous and factors through the homotopy category $H C, S=F \circ H$, where $H: C \rightarrow H C$ is the homotopy functor and $F: H C \rightarrow S C$. We will only be interested in the case in [6] that the projection functor $E: C \rightarrow E C$ is the homotopy functor $H$ and that the functor $S$ is the unique one given by Theorem 5.5 of [6] for $H$. For convenience, we will assume that $S$ is the functor of shape constructed in $\S 4$ of [6] for the pair $(C, H)$. We remark that the $H$-objects in this case are the compacta homotopically dominated by polyhedra [6, Example 5.9] which includes the ANR's. In an Appendix we show that the Holsztyński approach to shape in [6] and the approach of Mardešic and Segal in [8] using ANR-systems are equivalent so that our results apply to either theory.

Let $A$ and $B$ denote compact connected abelian topological groups. In the first section of this paper it is shown that if $G$ is a morphism in the shape category $S C$ between the continua $A$ and $B$, then there is a unique continuous homomorphism $g: A \rightarrow B$ such that $S(g)=G$. We obtain one immediate corollary: $A$ and $B$ are

Received by the editors October 19, 1972.

AMS (MOS) subject classifications (1970). Primary 55D99; Secondary 22 B99.

Key words and phrases. Shape, homotopy, movable compactum, shape domination, fundamental dimension, compact connected abelian topological group.

(1) This research was supported by NSF grant GP-24616A\#1. 
shape equivalent if and only if they are isomorphic as topological groups. In the next three sections of the paper we use the results of the first section to study the shape properties of continua which support abelian topological group structures. The properties that we study are movability, shape domination, and fundamental dimension.

Notation. We assume a knowledge of shape theory as developed in the paper of W. Holsztyński [6]. Except for the Appendix we are only interested in the case that the category $C$ is that of compact Hausdorff spaces and continuous maps and the projection functor $E$ in $\S \S 2,3,4$, and 5 of [6] is the homotopy functor $H$. We assume a knowledge of locally compact topological groups and of the structure of locally compact abelian topological groups. A knowledge of Pontryagin duality is also assumed. The second edition of Pontryagin's book [9] is a good reference. If $A$ is a locally compact abelian topological groups and $T$ is the circle group, then the character group of $A, \operatorname{Hom}(A, T)$ with the compact-open topology, is denoted by char $A$. If $g: A \rightarrow B$ is a continuous homomorphism, then the dual of $g$ is denoted by $g^{*}: \operatorname{char} B \rightarrow$ char $A$. If $G$ is a connected locally compact topological group, then there is an inverse system of Lie groups $\left\{L_{\alpha} ; \pi_{\alpha \beta} ; \alpha \leq \beta \in A\right\}$ with the projections $\pi_{\alpha}(G)=L_{\alpha}$ continuous homomorphisms. If $G$ is compact connected and abelian, then the $L_{\alpha}$ 's are tori $T_{\alpha}^{n_{\alpha}}$.

1. Shape morphisms and continuous homomorphisms. In this section we show that if $A$ and $B$ are compact connected abelian topological groups, then there is a one-to-one correspondence between the continuous homomorphisms from $A$ to $B$ and the morphisms between $A$ and $B$ as continua in the shape category. First we state a theorem essentially due to W. Scheffer [11].

1.1. Theorem (Scheffer). Let $A$ be a compact connected topological group and $B$ a compact connected abelian topological group with identities $e$ and 0 , respectively. If $f: A \rightarrow B$ is a continuous map with $f(e)$ in the arc-component of 0 in $B$, then there is a unique continuous homomorphism from $A$ to $B$ homotopic to $f$.

Observe that $f$ in the theorem is homotopic to a continuous $g: G \rightarrow B$ with $g(e)=0$. Theorem 1.1 then follows from Corollary 2 of [11].

We will use Theorem 1.1 to prove Theorem 1.2. Then taking 1.1 and 1.2 together we have a description of the relationship of the shape functor and the homotopy functor on continua supporting abelian group structures.

1.2. Theorem. Let $A$ and $B$ be compact connected abelian topological groups and let $G$ be a morphism in the shape category from $A$ to $B$. Then there is a unique continuous homomorphism $g: A \rightarrow B$ such that $S(g)=G$.

Proof. Let $G$ be a morphism between the compact spaces $A$ and $B$ in the shape category $S C$. Then $G$ is a covariant functor $G: H_{B} \rightarrow H_{A}$ such that $G(H(f))$ $=H(f)$ for each morphism $H(f)$ in $H_{B}$ (see $\left.[6, \S 4]\right)$. Recall that $H_{X}$ is the category which has as its objects the class of morphisms $H(p): X \rightarrow P$ in the homotopy category $H C$ where $P$ is an $H$-object in $C$. The morphisms of $H_{X}$ are 


$$
\operatorname{Mor}_{H_{X}}(H(p), H(q))=\{H(f): P \rightarrow Q: H(f) \circ H(p)=H(q)\}
$$

where $H(p): X \rightarrow P$ and $H(q): X \rightarrow Q$ are objects of $H_{X}$. Now if $T$ is the circle group, then the set of all homotopy classes of maps $\operatorname{Mor}_{H C}(A, T)$ and Mor $_{H C}(B, T)$ is a subclass of the objects of $H_{A}$ and $H_{B}$, respectively, since $T$ is an $H$-object. If we let + denote the group operation on $T$, then $\operatorname{Mor}_{H C}(A, T)$ and $\operatorname{Mor}_{H C}(B, T)$ have group structures induced by + which are isomorphic to char $A$ and char $B$ where the isomorphism from $\operatorname{Mor}_{H C}(A, T)$ and $\operatorname{Mor}_{H C}(B, T)$ to char $A$ and char $B$ is given by Theorem 1.1. Now $G$ : ob $H_{B} \rightarrow$ ob $H_{A}$ has the property that $G\left(\operatorname{Mor}_{H C}(B, T)\right) \subset \operatorname{Mor}_{H C}(A, T)$. This is because if $f: A \rightarrow P$ is a continuous map with $P$ an $H$-object and $G(H(f))=H(p)$, then $p: B \rightarrow P$; otherwise $G(H(i)) \neq H(i)$ where $i: P \rightarrow P$ is the identity map on $P$ with $H(i)$ a 1-2orphism in $H_{B}$. Now the group structure on $\operatorname{Mor}_{H C}(A, T)$ and $\operatorname{Mor}_{H C}(B, T)$ is induced by $+: T^{2} \rightarrow T$ with $T^{2}$ also an $H$-object. If $H(f)$ and $H(g)$ are in $\operatorname{Mor}_{H C}(B, T)$, then one can easily show that $G(H(f)+H(g))=G(H(f))$ $+G(H(g))$ using the fact that $G(H(+))=H(+)$. Therefore $G$ induces a homomorphism $G^{\prime}: \operatorname{Mor}_{H C}(B, T) \rightarrow \operatorname{Mor}_{H C}(A . T)$. This induces a homomorphism $G^{*}:$ char $B \rightarrow \operatorname{char} A$. Let $g: A \rightarrow B$ be the unique continuous homomorphism which induces $G^{*}:$ char $B \rightarrow \operatorname{char} A$. Now $g$ induces a shape morphism $S(g): A \rightarrow B$ which we will now show must be equal to $G$. Suppose that $S(g) \neq G$. As remarked in the Introduction, $B=\operatorname{inv} \lim \left\{T_{\alpha}^{n_{\alpha}} ; \pi_{\alpha \beta} ; \alpha \leq \beta\right.$ $\in A\}$ where the $T_{\alpha}^{n_{\alpha}}$ 's are tori and the $\pi_{\alpha \beta}$ 's are continuous homomorphisms. Let $\left\{\pi_{\alpha}: \alpha \in A\right\}$ be the projections making $B$ the projective limit of the inverse system. By [6, Lemma 4.1], there must be an $\alpha$ such that $S(g)\left(H\left(\pi_{\alpha}\right)\right)$ $\neq G\left(H\left(\pi_{\alpha}\right)\right)$ : Let $T_{\alpha}^{n_{\alpha}}=T_{1} \times \cdots \times T_{n_{\alpha}}$ and let $p_{i}: T_{\alpha}^{n_{\alpha}} \rightarrow T_{i}$ be the coordinate projections for $i=1, \ldots, n_{\alpha}$. Since $S(g)\left(H\left(\pi_{\alpha}\right)\right) \neq G\left(H\left(\pi_{\alpha}\right)\right)$, there must be an index $i$ such that $H\left(p_{i}\right) \circ S(g)\left(H\left(\pi_{\alpha}\right)\right) \neq H\left(p_{i}\right) \circ G\left(H\left(\pi_{\alpha}\right)\right)$. For that index $i$ we have that $H\left(p_{i}\right) \circ S(g)\left(H\left(\pi_{\alpha}\right)\right)=S(g)\left(H\left(p_{i} \circ \pi_{\alpha}\right)\right)$ and $H\left(p_{i}\right) \circ G\left(H\left(\pi_{\alpha}\right)\right)$ $=G\left(H\left(p_{i} \circ \pi_{\alpha}\right)\right)$. However, $H\left(p_{i} \circ \pi_{\alpha}\right) \in \operatorname{Mor}_{H C}(B, T)$ and thus $S(g)\left(H\left(p_{i}\right.\right.$ $\left.\left.\circ \pi_{\alpha}\right)\right)=G\left(H\left(p_{i} \circ \pi_{\alpha}\right)\right)$. This implies that $H\left(p_{i}\right) \circ S(g)\left(H\left(\pi_{\alpha}\right)\right)=H\left(p_{i}\right)$ - $G\left(H\left(\pi_{\alpha}\right)\right)$, a contradiction. Therefore $S(g)=G$.

If $g$ and $h$ are continuous homomorphisms from $A$ to $B$ such that $S(g)=S(h)$ $=G$, then $g^{*}=h^{*}=G^{*}: \operatorname{char} B \rightarrow$ char $A$. Thus $g=h$ and the continuous homomorphism $g$ is unique. The proof is complete.

1.3. Corollary. If $A$ and $B$ are compact connected abelian topological groups and have the same shape, then $A$ and $B$ are isomorphic as topological groups.

1.4. Example. In [1, p. 331], examples are given of compact connected Lie groups which have the same homotopy type (hence are of the same shape), but which are not isomorphic. Actually, the Lie groups can be taken to be homeomorphic and not isomorphic. Thus the fact that $A$ and $B$ are abelian in 1.2 and 1.3 is necessary. It is worth noting that $\mathrm{H}$. Scheerer has shown in [10] that two compact connected simply connected Lie groups having the same homotopy type are isomorphic. 
1.5. Example. Let $C$ be the Cantor set. It is well known that there are $2^{x_{0}}$ nonisomorphic abelian group structures on $C$ making $C$ a topological group. Thus it is necessary to have $A$ and $B$ connected in 1.2 and 1.3.

1.6. Remark. Theorems 1.1 and 1.2 taken together show the relationship between the homotopy and shape functors on $\operatorname{Mor}_{C}(A, B)$ where $A$ and $B$ are compact connected abelian topological groups. If $f, g: A \rightarrow B$ are continuous functions with $S(f)=S(g)$, then $H(f)=H(g)$ if and only if for some (or for all) $a \in A, f(a)$ and $g(a)$ are in the same arc-component of $B$.

1.7. Remark. There is another proof of Corollary 1.3. According to Steenrod [12], if $A$ is a compact connected abelian topological group, then there is an isomorphism from $H^{1}(A, Z)$ to char $A$ where $H^{1}(A, Z)$ is Čech cohomology with integer coefficients. Now Cech cohomology is a shape invariant. Thus if $A$ and $B$ are shape equivalent, then $H^{1}(A, Z) \simeq H^{1}(B, Z)$. Thus char $A \simeq \operatorname{char} B$ and $A \simeq B$. S. Godlewski showed Corollary 1.3 for solenoids [3].

2. Movability. In this section we show that a compact connected abelian topological group is movable if and only if it is locally connected. In this section of the paper we will assume that the reader is familiar with the Mardešic and Segal approach to shape using ANR-systems [8]. We use their definition of movability for a compactum $X$ in terms of an ANR-system associated with $X$. Movability is a shape invariant in the sense of Mardešic and Segal [7]. By the results in the Appendix it is a shape invariant in the sense of Holsztyński.

2.1. Definition. An ANR-system $\mathbf{X}=\left\{X_{\alpha} ; \pi_{\alpha \beta} ; \alpha \leq \beta \in A\right\}$ is said to be movable provided that for each $\alpha \in A$, there is a $\beta \geq \alpha$ such that for every $\gamma \geq \beta$ there is a map $r^{\beta \gamma}: X_{\beta} \rightarrow X_{\gamma}$ such that $H\left(\pi_{\alpha \gamma} \circ r^{\beta \gamma}\right)=H\left(\pi_{\alpha \beta}\right)$. A compactum $X$ is said to be movable if an ANR-system associated with it is movable. As shown in [7], if one ANR-system associated with $X$ is movable, then every one is and movability is a shape invariant.

2.2. Definition. Let $G$ be an abelian group and $H$ a subgroup of $G$. Then $H$ is said to admit division if whenever $g \in G$ and $n$ is a positive integer, then $n g \in H$ implies that $g \in H$. This is equivalent to saying that $G / H$ is torsion free. The group $G$ is said to have property $L$ if every finite subset of $G$ is contained in a finitely generated subgroup that admits division.

The following theorem is in Pontryagin [9, Theorem 48, p. 260].

2.3. Theorem. A compact abelian topological group $A$ is locally connected if and only if char $A$ has property $L$.

In the Introduction it was noted that it is known that a compact connected abelian topological group is the inverse limit of an inverse system of tori $\left\{T_{\alpha}^{n_{\alpha}} ; \pi_{\alpha \beta} ; \alpha \leq \beta \in B\right\}$ with onto bonding maps which are continuous homomorphisms and with projection maps $\pi_{\alpha}(A)=T_{\alpha}^{n_{\alpha}}$ which are continuous homomorphisms. We will now show that this inverse system can be taken to be an ANRsystem as in $[8, \S 2]$. The only thing that the system lacks is that the index set $B$ may not be closure finite and this is easily remedied. 
2.4. Lemma. Let $B$ be a directed set. Then there is a closure finite directed set $B^{\prime}$ and an increasing function $f: B^{\prime} \rightarrow B$ with $f\left(B^{\prime}\right)$ cofinal in $B$.

Proof. Let $\mathcal{Y}(B)$ be the set of all finite subsets of $B$ and let $B^{\prime}=\{F \in \mathcal{Y}(B)$ : there is an $a \in F$ with $\alpha \geq \beta$ for all $\beta \in F\}$. Let the order on $B^{\prime}$ be defined by $F \geq F^{\prime}$ if and only if $F \supset F^{\prime}$. Now $B^{\prime}$ is a directed set since for $F_{1}$ and $F_{2}$ in $B^{\prime}$, there is an $\alpha \in B$ with $\alpha \geq \beta$ for all $\beta \in F_{1} \cup F_{2}$. If we let $F_{3}=\{\alpha\} \cup F_{1}$ $\cup F_{2}$, then $F_{3} \geq F_{1}$ and $F_{3} \geq F_{2}$ with $F_{3} \in B^{\prime}$. It is closure finite since if $F \in B^{\prime}$, then there are less than $2^{\text {card } F} G$ 's in $B^{\prime}$ with $G \leq F$. Let $f: B^{\prime} \rightarrow B$ be any function having the property that $f(F)=\alpha$ where $\alpha \in F$ and $\alpha \geq \beta$ for all $\beta \in F$. Then if $F \geq F^{\prime}$, then $f(F) \geq f\left(F^{\prime}\right)$ and $f$ is an increasing function. Actually $f\left(B^{\prime}\right)$ is not only cofinal, it is onto since $\{\alpha\} \in B^{\prime}$ for all $\alpha \in B$ and $f(\{\alpha\})=\alpha$.

Now we can make $\left\{T_{\alpha}^{n_{\alpha}} ; \pi_{\alpha \beta} ; \alpha \leq \beta \in B\right\}$ into an ANR-system by replacing $B$ by the $B^{\prime}$ given in Lemma 2.4 and obtaining $\left\{T_{F}^{n_{F}} ; \pi_{F G} ; F \leq G \in B^{\prime}\right\}$ where $T_{F}^{n_{F}}=T_{f(F)}{ }^{n_{f(F)}}$ and $\pi_{F G}=\pi_{f(F) f(G)}$. Then $A$ is the projective limit of this ANRsystem with projection maps $\pi_{F}=\pi_{f(F)}$.

We also remark that Lemma 2.4 shows that there is no topological significance to Theorem 7 in [8] since every compact Hausdorff space is known to be the inverse limit of compact ANR's. We can now state and prove the main theorem in this section.

2.5. Theorem. Let $A$ be a compact connected abelian topological group. Then $A$ is movable if and only if char $A$ has property $L$. Consequently, $A$ is movable if and only if $A$ is locally connected.

Proof. First we need some preliminary remarks. Now char $A$ is the direct limit of its finitely generated subgroups. That is, let $\left\{H_{\alpha} ; \pi_{\alpha \beta} ; \alpha \leqslant \beta \in B\right\}$ be such that $\alpha \leqslant \beta$ if and only if $H_{\alpha} \subset H_{\beta}$ with $H_{\alpha}$ and $H_{\beta}$ finitely generated subgroups of char $A$ and $e_{\alpha \beta}: H_{\alpha} \rightarrow H_{\beta}$ the inclusion maps. Then the injection maps $e_{\alpha}: H_{\alpha} \rightarrow \operatorname{char} A$ make char $A$ the injective limit of the direct system $\left\{H_{\alpha} ; e_{\alpha \beta} ; \alpha \leqslant \beta \in B\right\}$. Since $A$ is connected, char $A$ is torsion free and each $H_{\alpha}$ is just a free group $Z_{\alpha}^{n}$. Taking the dual we get an inverse system $\left\{T_{\alpha}^{n_{\alpha}}=\operatorname{char} Z_{\alpha}^{n_{\alpha}} ; \pi_{\alpha \beta}=e_{\alpha \beta}^{*} ; \alpha \leq \beta \in B\right\}$ with $A$ the projective limit of the system by the projection maps $\pi_{\alpha}: A \rightarrow T_{\alpha}^{n_{\alpha}}$ being the unique continuous homomorphisms with $\pi_{\alpha}^{*}=e_{\alpha}$. Let $B^{\prime}$ be the closure finite directed set described in the proof of Lemma 2.4 associated with $B$ and let $\left\{T_{F}^{n_{F}} ; \pi_{F G} ; F \leq G \in B^{\prime}\right\}$ be the associated ANR-system. Now we will show that if char $A$ has property $\mathrm{L}$, then $A$ is movable. Suppose that char $A$ has property L. We will show that the ANR-system described above is movable and thus that $A$ is movable. Let $F=\left\{\alpha_{1}, \ldots, \alpha_{n}\right\} \in B^{\prime}$ with $f(F)=\alpha$. Then $T_{F}^{n_{F}}=T_{\alpha}^{n_{\alpha}}$. Let $Z_{\alpha}^{n_{\alpha}} \subset Z_{\beta}^{n_{\beta}}$ be such that $Z_{\beta}^{n_{\beta}}$ admits division in char $A$ using the fact that char $A$ has property L. Let $G=\left\{\beta, \alpha_{1}, \ldots, \alpha_{n}\right\} \in B^{\prime}$. Then $G \geq F$ and $f(G)=\beta$. Now suppose that $P \geq G$ in $B^{\prime}$. Since $Z_{G}^{n_{G}}$ admits division in char $A$, it admits division in $Z_{P}^{n_{P}}$. Thus $Z_{P}^{n_{P}} / Z_{G}^{n_{G}}$ is torsion free and since it is finitely generated it is a free group $Z^{q}$. Therefore $Z_{P}^{n_{P}} \simeq Z^{q} \times Z_{G}^{n_{G}}$. Define $r^{G P}: T_{G}^{n_{G}} \rightarrow T_{P}^{n_{P}}$ as the map which 
is the dual of the map from $Z_{P}^{n_{P}}$ to $Z_{G}^{n_{G}}$ having $Z^{q}$ as its kernel. Then we have $\pi_{F P} \circ r^{G P}=\pi_{F G}$. Thus $A$ is movable.

We will now show that if $A$ is movable, then char $A$ has property L. Suppose that $A$ is movable. We will use this fact to show that char $A$ has property L. Let $F \in B^{\prime}$ and let $G \geq F$ be as in the definition of movability. Suppose that $n$ is a positive integer and that $n a \in Z_{F}^{n_{F}}$ for some $a \in$ char $A$. Then we claim that $a \in Z_{G}^{n_{G}}$. To prove that let $P \geq G$ be such that $Z_{P}^{n_{P}}$ is the subgroup of char $A$ generated by $\{a\} \cup Z_{G}^{n_{G}}$. From the definition of movability and the choice of $G$, there is a continuous map $r^{G P}: T_{G}^{n_{G}} \rightarrow T_{P}^{n_{P}}$ such that $\pi_{F P} \circ r^{G P}$ is homotopic to $\pi_{F G}$. By Theorem 1.1, we may assume that $r^{G P}$ is a homomorphism and that $\pi_{F P} \circ r^{G P}=\pi_{F G}$. Thus we have a homomorphism $\rho=\left(r^{G P}\right)^{*}: Z_{P}^{n_{P}} \rightarrow Z_{G}^{n_{G}}$ such that $e_{F G}=\rho \circ e_{G P}$. Now $\rho(n a)=n a$ since $e_{F G}(n a)=n a$ and $e_{G P}(n a)=n a$. However, $n \rho(a)=\rho(n a)=n a$ with $\rho(a) \in Z_{G}^{n_{G}}$. Now $n$th roots are unique in char $A$ since char $A$ is torsion free. Thus $\rho(a)=a$ and $a \in Z_{G}^{n_{G}}$. Thus we have proved our claim that if $n a \in Z_{F}^{n_{F}}$, then $a \in Z_{G}^{n_{G}}$. Now let $L$ be the subgroup of $Z_{G}^{n_{G}}$ consisting of the elements $a \in Z_{G}^{n_{G}}$ such that for some integer $n, n a \in Z_{F}^{n_{F}}$. Then $L$ admits division in char $A$ and is finitely generated. Thus char $A$ has property $\mathrm{L}$.

2.6. Example. It is known that the solenoids are not movable. Theorem 2.5 implies this and allows us to construct examples of both movable and nonmovable continua by taking the character groups of discrete torsion free abelian groups having property $\mathrm{L}$ or not having property $\mathrm{L}$, respectively.

2.7. Theorem. Let $A$ be a compact connected abelian topological group. When $A$ is not movable it is not arcwise connected.

Proof. If $A$ is not movable, then char $A$ does not have property L. Let $Z^{n}$ be a finitely generated subgroup of char $A$ which is not contained in any finitely generated subgroup which admits division in char $A$. Let $M$ be the smallest subgroup of char $A$ containing $Z^{n}$ which does admit division in char $A$. Then the rank of $M$ is $n$ and $M$ does not have property $\mathrm{L}$. The imbedding homomorphism $e: M \rightarrow \operatorname{char} A$ induces a map $e^{*}: A \rightarrow$ char $M$ which is onto. Now the dimension of char $M$ is $n[9$, Theorem 47, p. 259] and char $M$ is not locally connected. Using the results of [2], char $M$ cannot be arcwise connected since it is finite dimensional and not locally arcwise connected. But $e^{*}(A)=$ char $M$ and thus $A$ cannot be arcwise connected either.

2.8. Example. Let $Z$ denote the integers. Let $A$ denote the compact connected abelian topological group which is the character group of the discrete group $Z^{x_{0}}$. By Theorem 2.5, $A$ is a movable continuum. It is known, however, that $A$ is not arcwise connected. In a subsequent paper we will show that any continuum shape equivalent to $A$ cannot be arcwise connected. Thus, the shape class of $A$ is movable, contains locally connected continua, but contains no arcwise connected continuum.

3. Shape domination. Let $X$ and $Y$ be compacta. Then $X$ shape dominates $Y$, 
$S(X) \geq S(Y)$, if and only if there are shape morphisms $G: X \rightarrow Y$ and $G^{\prime}: Y \rightarrow X$ such that $G \circ G^{\prime}$ is the identity shape morphism on $Y$. Theorem 1.2 allows us to prove the following theorem quite easily.

3.1. Theorem. Let $A$ and $B$ be compact connected abelian topological groups. Then $S(A) \geq S(B)$ if and only if there is a compact connected abelian topological group $C$ such that $A \simeq B \times C$.

Proof. It is clear that if $Z \simeq B \times C$, then $S(A) \geq S(B)$. Now suppose that $S(A) \geq S(B)$. Then by Theorem 1.2 there are continuous homomorphisms $g: A \rightarrow B$ and $h: B \rightarrow A$ such that $g \circ h: B \rightarrow B$ is the identity map. These homomorphisms induce homomorphisms $h^{*}: \operatorname{char} A \rightarrow \operatorname{char} B$ and $g^{*}: \operatorname{char} B$ $\rightarrow$ char $A$ with $h^{*} \circ g^{*}:$ char $B \rightarrow$ char $B$ the identity homomorphism. Now this implies that char $A \simeq \operatorname{char} B \oplus \operatorname{ker} h^{*}$. Letting $C=\operatorname{char} \operatorname{ker} h^{*}$ we get that $A \simeq B \times C$ and the theorem is proved.

4. Shape dimension. Let $X$ be a compactum. The fundamental dimension of $X$, $\mathrm{Sd}(X)$, is the minimum Lebesgue dimension of a compactum $Y$ such that $S(Y) \geq S(X)$. Clearly $\operatorname{Sd}(X) \leq \operatorname{dim} X$.

4.1. Theorem. Let $G$ be a compact connected topological group. Then $\operatorname{Sd}(G)$ $=\operatorname{dim} G$.

Proof. According to the remarks in the Introduction $G$ is the inverse limit of an inverse system of Lie groups, $\left\{L_{\alpha} ; \pi_{\alpha \beta} ; \alpha \leq \beta \in A\right\}$ with the $L_{\alpha}$ 's Lie groups and the projection maps, continuous surjective homomorphisms $\pi_{\alpha}: G \rightarrow L_{\alpha}$. By $\left[4, I .2 .11\right.$, p. 302] the homomorphisms in Čech cohomology $\pi_{\alpha}^{*}: H^{n}(L, Z)$ $\rightarrow H^{n}(G, Z)$ are nonzero for $n=\operatorname{dim} L_{\alpha}$. Now if $H^{n}(G, Z) \neq 0$ for an infinite number of $n$ 's, then $\operatorname{dim} G=\infty$. Also, $H^{n}(Y, Z) \neq 0$ for an infinite number of $n$ 's for any $Y$ shape dominating $G$ and thus $\operatorname{dim} Y=\infty$ also. Therefore $\operatorname{Sd}(G)=\operatorname{dim} G=\infty$. If $H^{n}(G, Z)=0$ for all $n>m$ with $H^{m}(G, Z) \neq 0$, then $\operatorname{dim} L_{\alpha} \leq m$ for all $\alpha \in A$. Thus $\operatorname{dim} G \leq m$. However, $\operatorname{dim} Y \geq m$ for any $Y$ with $S(Y) \geq S(G)$ since $H^{m}(Y, Z) \neq 0$. Thus $\operatorname{dim} G=\operatorname{Sd}(G)=m$ in this case also.

Appendix: The equivalence of two approaches to shape theory. Here we will prove that, for the category $C$ of compact Hausdorff spaces and continuous maps and the homotopy functor $H: C \rightarrow H C$, the shape functor $S: C \rightarrow S C$ given in [6] gives the same shape classification for compacta as the approach by Mardešic and Segal [8] using ANR-systems. Actually, the situation is more general as we will point out in a remark at the end. Let $X$ and $Y$ be compact Hausdorff spaces. Let $\mathbf{X}=\left\{X_{\alpha} ; \pi_{\alpha \beta} ; \alpha \leq \beta \in A\right\}$ and $\mathbf{Y}=\left\{Y_{\gamma} ; \pi_{\gamma \delta} ; \gamma \leq \delta \in \Gamma\right\}$ be ANR-systems associated with $X$ and $Y$, respectively, with $\left\{\pi_{\alpha}: X \rightarrow X_{\alpha}\right\}$ and $\left\{\rho_{\gamma}: Y \rightarrow Y_{\gamma}\right\}$ the projection maps making $X$ and $Y$ the projective limits of $\mathbf{X}$ and $\mathbf{Y}$, respectively.

Theorem. Let $\mathbf{f}: \mathbf{X} \rightarrow \mathbf{Y}$ be a map of ANR-systems. Then

(1) f induces a morphism $G=G(f)$ from $X$ to $Y$ in the shape category $S C$ 
uniquely defined by $G\left(H\left(\rho_{\gamma}\right)\right)=H\left(f_{\gamma} \circ \pi_{f(\gamma)}\right)$ for all $\gamma \in \Gamma$.

(2) The maps of ANR-systems $\mathbf{f}$ and $\mathbf{g}$ are homotopic if and only if $G(\mathbf{f})$ $=G(\mathrm{~g})$.

(3) If $G$ is a morphism from $X$ to $Y$ in $S C$, then there is a map of ANR-systems $\mathbf{f : ~} \mathbf{X} \rightarrow \mathbf{Y}$ with $G(\mathbf{f})=G$.

Proof. First we will show (1). Let $P$ be an $H$-object and $p: Y \rightarrow P$ a continuous function. There is a $\gamma \in \Gamma$ and a continuous map $p^{\gamma}: Y_{\gamma} \rightarrow P$ such that $H\left(p^{\gamma} \circ \rho_{\gamma}\right)=H(p)$. Then define $G(\mathbf{f})(H(p))=H\left(p^{\gamma} \circ f_{\gamma} \circ \pi_{f(\gamma)}\right)$. We will now show that $G(f)$ is well defined. Suppose that $\delta \in \Gamma$ and $p^{\delta}$ is such that $H\left(p^{\delta} \circ \rho_{\delta}\right)=H(p)$. Then there is a $\lambda \in \Gamma$ with $\lambda \geq \delta$ and $\lambda \geq \gamma$. Then $H(p)=H\left(p^{\gamma} \circ \rho_{\gamma \lambda} \circ \rho_{\lambda}\right)=H\left(p^{\delta} \circ \rho_{\delta \lambda} \circ \rho_{\lambda}\right)$. Thus there is a $\lambda^{\prime} \geq \lambda$ such that

$$
H\left(p^{\gamma} \circ \rho_{\gamma \lambda} \circ \rho_{\lambda \lambda^{\prime}}\right)=H\left(p^{\delta} \circ \rho_{\delta \lambda} \circ \rho_{\lambda \lambda^{\prime}}\right) .
$$

This implies that

$$
H\left(p^{\gamma} \circ \pi_{f(\gamma) f(\lambda)} \circ \pi_{f(\lambda) f\left(\lambda^{\prime}\right)}\right)=H\left(p^{\delta} \circ \pi_{f(\delta) f(\lambda)} \circ \pi_{f(\lambda) f\left(\lambda^{\prime}\right)}\right) .
$$

Thus,

$$
H\left(p^{\gamma} \circ \pi_{f(\lambda) f\left(\lambda^{\prime}\right)} \circ \pi_{f\left(\lambda^{\prime}\right)}\right)=H\left(p^{\delta} \circ \pi_{f(\delta) f\left(\lambda^{\prime}\right)} \circ \pi_{f\left(\lambda^{\prime}\right)}\right)
$$

and we have that $H\left(p^{\gamma} \circ \pi_{f(\gamma)}\right)=H\left(p^{\delta} \circ \pi_{f(\delta)}\right)$ and thus $G(f)(H(p))$ is well defined. If we define $G(f)(H(g))=H(g)$ for $H(g)$ a morphism in $H_{Y}$, then $G(f)$ becomes a covariant functor from $H_{Y}$ to $H_{X}$ having the property that $G\left(H\left(\rho_{\gamma}\right)\right)$ $=H\left(f_{\gamma} \circ \pi_{f(\gamma)}\right)$ for all $\gamma \in \Gamma$. According to Lemma 4.1 of [6], this defines $G$ uniquely.

Now we will prove (2). First suppose that $\mathbf{f}$ and $\mathbf{g}$ are homotopic as maps of ANR-systems. We will show that $G(\mathbf{f})=G(\mathrm{~g})$. Let $\gamma \in \Gamma$. Since $G\left(H\left(\rho_{\gamma}\right)\right)$ $=H\left(f_{\gamma} \circ \pi_{f(\gamma)}\right)$ uniquely defines $G(\mathrm{f})$ we need only show that $G(f)\left(H\left(\rho_{y}\right)\right)$ $=G(\mathrm{~g})\left(H\left(\rho_{\gamma}\right)\right)$. By the definition of $\mathbf{f}$ and $\mathbf{g}$ being homotopic [8, p. 43] we have that there is an $\alpha \in A$ with $\alpha \geq f(\gamma)$ and $\alpha \geq g(\gamma)$ such that

$$
H\left(f_{\gamma} \circ \pi_{f(\gamma) \alpha}\right)=H\left(g_{\gamma} \circ \pi_{g(\gamma) \alpha}\right) .
$$

This implies that

$$
H\left(f_{\gamma} \circ \pi_{f(\gamma) \alpha} \circ \pi_{\alpha}\right)=H\left(g_{\gamma} \circ \pi_{g(\gamma) \alpha} \circ \pi_{\alpha}\right)
$$

and therefore that

$$
H\left(f_{\gamma} \circ \pi_{f(\gamma)}\right)=H\left(g_{\gamma} \circ \pi_{g(\gamma)}\right)
$$

Thus

$$
G(\mathbf{f})\left(H\left(\rho_{\gamma}\right)\right)=G(\mathbf{g})\left(H\left(\rho_{\gamma}\right)\right) \text { and } G(\mathbf{f})=G(\mathbf{g}) \text {. }
$$

Now suppose that $G(\mathbf{f})=G(\mathrm{~g})$. Let $\gamma \in \Gamma$. Then $H\left(f_{\gamma} \circ \pi_{f(\gamma)}\right)=H\left(g_{\gamma} \circ \pi_{g(\gamma)}\right)$. 
Let $\alpha \in A$ with $\alpha \geq f(\gamma)$ and $\alpha \geq g(\gamma)$. Then

$$
H\left(f_{\gamma} \circ \pi_{f(\gamma) \alpha} \circ \pi_{\alpha}\right)=H\left(g_{\gamma} \circ \pi_{g(\gamma) \alpha} \circ \pi_{\alpha}\right) .
$$

By Property (2.3) in [6, p. 160] (or by Lemma 4 of [8]), there is a $\beta \geq \alpha$ with

$$
H\left(f_{\gamma} \circ \pi_{f(\gamma) \alpha} \circ \pi_{\alpha \beta}\right)=H\left(g_{\gamma} \circ \pi_{g(\gamma) \alpha} \circ \pi_{\alpha \beta}\right) .
$$

That is, $H\left(f_{\gamma} \circ \pi_{f(\gamma) \beta}\right)=H\left(g_{\gamma} \circ \pi_{g(\gamma) \beta}\right)$ with $\beta \geq f(\gamma)$ and $\beta \geq g(\gamma)$. This implies that $\mathbf{f}$ and $\mathbf{g}$ are homotopic as maps of ANR-systems.

Now we will show (3). Let $G$ be a shape morphism from $X$ to $Y$ which is a covariant functor from $H_{Y}$ to $H_{X}$ as in [6, §4]. Then, for $\gamma \in \Gamma$, let $g_{\gamma}: X \rightarrow Y_{\gamma}$ be such that $G\left(H\left(\rho_{\gamma}\right)\right)=H\left(g_{\gamma}\right)$. Because $G(H(p))=H(p)$ for every morphism $H(p)$ in $H_{Y}\left[6\right.$, p. 162] we have that, for $\gamma \geq \delta, H\left(g_{\gamma}\right)=H\left(\rho_{\gamma \delta}\right) \circ H\left(g_{\delta}\right)$. By Remark 2 of $[8$, p. 51] we get a map of ANR-systems $\mathbf{f}: \mathbf{X} \rightarrow \mathbf{Y}$ such that $H\left(f_{\gamma} \circ \pi_{f(\gamma)}\right)=G\left(H\left(\rho_{\gamma}\right)\right)$. Then, by (1), $G(\mathbf{f})=G$. The proof of the theorem is now complete.

Corollary. Two compacta are shape equivalent in the sense of Holsztyniski if and only if they are shape equivalent in the sense of Mardešic and Segal.

There is a way to generalize the approach of Mardešic and Segal. Let $E: C \rightarrow E C$ be a projection functor on a semiclassical $E$-category $C$. Let $A(C)$ denote the category of $E$-object systems where the objects of $A(C)$ are inverse systems $\mathbf{X}=\left\{X_{\alpha} ; \pi_{\alpha \beta} ; \alpha \leq \beta \in A\right\}$ of $E$-objects $X_{\alpha}$ in $C$ where the directed set $A$ is closure finite. Let $\mathbf{Y}=\left\{Y_{\gamma} ; \pi_{\gamma \delta} ; \gamma \leq \delta \in \Gamma\right\}$ be another $E$-object system. Let a map of $E$-object systems $\mathbf{f}: \mathbf{X} \rightarrow \mathbf{Y}$ be defined by an increasing function $f: \Gamma \rightarrow A$ and morphisms $f_{\gamma}: X_{f(\gamma)} \rightarrow Y_{\gamma}$ in $C$ for each $\gamma \in \Gamma$ satisfying the condition that, for $\gamma \leq \delta$,

$$
E\left(f_{\gamma} \circ \pi_{f(\gamma) f(\delta)}\right)=E\left(\rho_{\gamma \delta} \circ f_{\delta}\right)
$$

Define two maps of $E$-object systems to be homotopic in a manner analogous to maps of ANR-systems [8, p. 43].

Let $X$ be an object in $C$. Then $X$ is associated with the $E$-object system $\mathbf{X}$ if there is a presentation of $X,\left\{X ; \pi_{\alpha} ; \alpha \in A\right\}$ making $X$ the projective limit of the inverse system $\mathbf{X}$ in $C$. By the definition of an $E$-category and by Lemma 2.4 of this paper, every object $X$ in $C$ is associated with an $E$-object system. Except for the fact that $A$ is closure finite, the only properties of ANR-systems used in the proof of the previous theorem in this Appendix are the properties in Lemma 3 and Lemma 4 in [8] which are properties (2.2) and (2.3) of E-objects in [6]. The proof of the previous theorem can be modified slightly to give a proof of the following theorem where $X$ and $Y$ are objects of $C$ associated with the $E$-object systems $\mathbf{X}$ and $\mathbf{Y}$. The functor of shape $S: C \rightarrow S C$ is that given in $\S \S 4$ and 5 of [6] for the functor $E$. 
Theorem. Let $\mathbf{f}: \mathrm{X} \rightarrow \mathrm{Y}$ be a map of E-object systems. Then (1) $\mathbf{f}$ induces a morphism $G=G(f)$ from $X$ to $Y$ in the shape category $S C$ uniquely defined by $G\left(E\left(\rho_{\gamma}\right)\right)=E\left(f_{\gamma} \circ \pi_{f(\gamma)}\right)$ for all $\gamma \in \Gamma$.

(2) The maps of E-object systems $\mathbf{f}$ and $\mathbf{g}$ are homotopic if and only if $G(\mathbf{f})=G(\mathbf{g})$.

(3) If $G$ is a morphism from $X$ to $Y$ in $S C$, then there is a map of E-object systems $\mathrm{f:} \mathbf{X} \rightarrow \mathbf{Y}$ with $G(\mathbf{f})=G$.

Thus, for such a category $C$ and projection functor $E$, these developments lead to the same notion of shape equivalence.

\section{REFERENCES}

1. P.F. Baum and W. Browder, The cohomology of quotients of classical groups, Topology 3 (1965), 305-336. MR 32 \#6490.

2. A.M. Gleason and R.S. Palais, On a class of transformation groups, Amer. J. Math. 79 (1957), 631-648. MR 19, 663.

3. S. Godlewski, On the shape of solenoids, Bull. Acad. Polon. Sci. Sér. Sci. Math. Astronom. Phys. 17 (1969), 623-627. MR 41 \#9252.

4. K.H. Hofmann and P.S. Mostert, Elements of compact semigroups, Charles E. Merrill Books, Columbus, Ohio, 1966. MR 35 \#285.

5. W. Holsztyński, Continuity of Borsuk's shape functor, Bull. Acad. Polon. Sci. Sér. Sci. Math. Astonom. Phys. 19 (1971), 1105-1108.

6. - An extension and axiomatic characterization of Borsuk's theory of shape, Fund. Math. 70 (1971), no. 2, 157-168. MR 43 \#8080.

7. S. Mardešíc and J. Segal, Movable compacta and ANR-systems, Bull. Acad. Polon. Sci. Sér. Sci. Math. Astronom. Phys. 18 (1970), 649-654. MR 44 \# 1026.

8. - Shapes of compacta and ANR-systems, Fund. Math. 72 (1971), no.1, 41-59. MR 45 \#686.

9. L.S. Pontrjagin, Continuous groups, 2nd ed., GITTL, Moscow, 1954; English transl., Gordon and Breach, New York, 1966. MR 17, 171; 34 \# 1439.

10. H. Scheerer, Homotopiedquivalente kompakte Liesche Gruppen, Topology 7 (1968), 227-232. MR 37 \# 4833.

11. W. Scheffer, Maps between topological groups that are homotopic to homomorphisms, Proc. Amer. Math. Soc. 33 (1972), 562-567.

12. N. Steenrod, Universal homology groups, Amer. J. Math. 58 (1936), 661-701.

DEPARTMENT OF MATHEMATICS, UNIVERSITY OF FLORIDA, GAINESVILLE, FLORIDA 32601 